\title{
Uma proposta de modelagem matemática no ensino-aprendizagem de matrizes
}

\author{
Ademir Costa $\quad$ Thiago Lopes
}

\begin{abstract}
Resumo
No presente trabalho de relato de experiência foram desenvolvidas situações-problemas reais envolvendo o estudo e aplicação de matrizes, utilizando-os desde a economia doméstica até a mais simples organização de dados em tabelas. Propõe-se, baseada em recomendações do professor, que os alunos façam uma pesquisa exploratória no comércio local, como postos de combustíveis, farmácias, açougues, nos comércios e mercados em geral, para investigarem os preços de custo e preços de venda de alguns produtos. Desse modo os alunos se confrontam com a questão de pesquisar e tendo assim que sair de dentro da sala de aula para fazer a coleta de dados. Participaram deste trabalho 34 alunos do $2^{\mathrm{o}}$ ano do ensino médio, turma "A", da Escola Estadual de Ensino Médio José Luiz Martins, no município de Água Azul do Norte no estado do Pará. Através da metodologia da modelagem Matemática, descreveremos aqui, uma experiência de sala e extra sala de aula.
\end{abstract}

Palavras-chave: Educação; Modelagem; Matrizes; Ensino; Aprendizagem.

\section{Abstract}

In the present work of experience reporting, real problem situations were developed involving the study and applications of matrices, from the domestic economy to the simplest organization of data in tables. It is proposed, based on recommendations of the teacher, that students conduct an exploratory research in local commerce, such as gas stations, pharmacies, butchers, in the trade and markets in general, to investigate the cost and sales prices of some products. In this way the students are confronted with the question of researching and having thus to leave the classroom to make the data collection. Participated in this study 34 students from the 2nd year of high school, group "A", from the State School of Higher Education José Luiz Martins, in the city of Água Azul do Norte in the state of Pará. Through the mathematical modeling methodology, we describe here an experience of room and extra-classroom.

Keywords: Education; Modeling; Matrices; Teaching; Learning.

\section{Introdução}

Se fizéssemos uma viagem no tempo, observaríamos que as transformações e mudanças sociais dependem única e exclusivamente das necessidades do meio ao qual elas estão inseridas. O desenvolvimento das ciências, e mais especificamente da Matemática, no decorrer de todo esse tempo, 
não foi diferente. As sociedades cresciam e com esse crescimento nascia o desejo de desenvolver-se cultural, econômica e tecnologicamente.

O ensino é o meio fundamental para tais transformações ocorrerem. E é papel da escola, compartilhar os conhecimentos desenvolvidos no meio científico. Ferreira e Wodewotzki ([10]) afirmam que "tais transformações aprofundam uma exclusão social, desafiando assim o ambiente de ensino. A escola deve considerar e buscar atender tais desafios.". Porém, esse compartilhar de informações deve ser significativo, para que essa exclusão social seja cada vez mais amenizada.

Nos dias atuais, o ensino de Matemática persiste sendo vilão no aprendizado dos estudantes, pois depende muito da atuação dos dois principais atores da real eficácia do ensino-aprendizagem, o professor e o aluno. No contexto do desinteresse em relação à aula de Matemática e a exclusão que a própria disciplina acaba perpetrando, constatamos em [8], [12] e [15] que tais rejeições ao estudo da Matemática decorre muitas vezes da padronização das aulas, sempre constante, falta de inovação e atrativos e a não significância do conteúdo aplicado para com a realidade dos alunos. Assim, podemos perceber que para haver aprendizagem significativa...,

...são necessárias duas condições. Em primeiro lugar, o aluno precisa ter uma disposição para aprender: se o indivíduo quiser memorizar o conteúdo arbitrária e literalmente, então a aprendizagem será mecânica. Em segundo, o conteúdo escolar a ser aprendido tem que ser potencialmente significativo, ou seja, ele tem que ser lógico e psicologicamente significativo: o significado lógico depende somente da natureza do conteúdo, e o significado psicológico é uma experiência que cada indivíduo tem. Cada aprendiz faz uma filtragem dos conteúdos que têm significado ou não para si próprio. [13, p38]

Assim, como citado acima, o interesse do aluno é primordial para o bom desenvolvimento do trabalho, Pelizzari et al. ([13]) e Burak ([7]) fundamentam essas ideias, reforçando que o aluno deve estar motivado para receber a gama de informações que a escola formal lhe oferecerá. Contudo, o professor deve mostrar-lhe que todo aquele conteúdo desenvolvido nas aulas, isto é, aquilo que pretendemos que ele aprenda, será de suma importância para o seu desenvolvimento de modo geral. Sendo assim, o professor deverá identificar o conhecimento prévio do aluno para formar um plano de aula significativo e apto a intervenções e mudanças durante o processo de ensino-aprendizagem.

Aquilo que o educando já sabe, o conhecimento prévio (conceitos, proposições, teoremas, axiomas, princípios, imagens, símbolos, enfim, tudo o que ele já conhece), é essencial para a teoria da aprendizagem significativa, pois constitui-se como a essência do processo de aprendizagem, uma vez que é significativo por definição, basilar na transformação dos significados lógicos dos materiais de aprendizagem, potencialmente significativos, em significados psicológicos ([2], [3]).

Entretanto, acreditamos que a proposta apresentada neste artigo, traz em seu bojo, um modelo significativo no ensino-aprendizagem de matrizes, pois se apoia na metodologia denominada Modelagem Matemática, que vem crescendo e se desenvolvendo com uma nova visão de Educação Matemática, que valoriza não somente a aquisição de conhecimentos, mas o desenvolvimento de competências, caráteres e valores, relacionando a Matemática com o mundo real.

Atualmente, nós professores, temos o grande desafio de atrair o aluno para o universo onde a Matemática é o instrumento fundamental para resolução dos problemas cotidianos, problemas que transcendem qualquer ramo do conhecimento humano. Devemos atraí-los, para uma Matemática 
dinâmica, reflexiva e crítica, que ele próprio, "através da investigação, da descoberta e da validação dos resultados, aponte caminhos para compreensão da realidade social, e com possibilidades de atuar sobre ela e que atenda também às gerações futuras." [10, p126]. Assim, contribuiríamos com um ensino significativo e também garantiríamos uma aprendizagem de qualidade. Faríamos com que os alunos adquirissem interesse pelo estudo da Matemática, pois o grande desafio de qualquer professor é levar a Matemática para o contexto de vivência do estudante. Essa barreira que separa ensino significativo de aprendizado de qualidade pode ser rompida com o apoio da modelagem. De tal modo, apresentamos aqui uma experiência vivida com alunos do $2^{\mathrm{o}}$ ano do ensino médio, apoiados na metodologia de modelagem matemática para o ensino de matrizes.

Os estudos de matrizes se justificam por contribuir com os avanços científicos e tecnológicos, destacando-se nos campos mais variados como: na engenharia, na informática, na administração, na economia, dentre outros segmentos que possam envolver organização de dados em tabelas. O ensino de matrizes traz consigo as ideias de estrutura, além de constituir uma ferramenta que auxilia na resolução dos sistemas lineares. Sendo muito útil no ensino de Matemática de nível básico como um importante instrumento, tanto no uso em seu cotidiano quanto na Álgebra Linear estudada na Matemática do ensino superior.

Pensando em todo esse contexto, nossa preocupação em apresentar essa proposta de ensino, é relacionar esse conteúdo matemático com o meio social dos alunos. Para tanto, os alunos devem tornarse pesquisadores, para que os mesmos sintam-se inseridos nesse processo de ensino-aprendizagem. Mostrando a eles, que além de fazerem parte, são o próprio processo, pois são eles que identificam, pesquisam e materializam o problema gerindo modelos matemáticos esquematizados e estruturados.

\section{O problema e a proposta de modelagem}

A Modelagem Matemática é uma metodologia diferenciada que direciona o estudante ao campo da pesquisa, instigando o mesmo a ir em busca do conhecimento, tornando as aulas mais interessantes e atraentes, relacionando o conhecimento escolar com o contexto do aluno, motivando e preparando-os para utilizarem a Matemática em distintas circunstâncias.

D’ambrosio (1998), Bassanezi ([6]) e Barbosa ([5]) sinalizam, além das características mencionadas acima, a Modelagem Matemática como fio condutor que liga e conecta o ensino de conteúdos de Matemática com outras formas de conhecimento. Essas ideias confirmam que devemos sair da rotina de sala de aula e contemplar os estudantes com problemas desafiadores e significativos ao invés de situações padronizadas e reproduzidas.

A proposta se baseia na recomendação do professor aos alunos que façam uma pesquisa exploratória no comércio local, como postos de combustíveis, mercados, farmácias, açougues, frutarias, entre outros, para averiguar o preço de custo e preço de venda de alguns produtos. Desse modo os alunos se confrontam com a questão de pesquisar e tendo assim que sair de dentro da sala de aula para fazer a coleta de dados. Nessa perspectiva, os alunos teriam grande responsabilidade na execução da atividade desenvolvendo-a do início ao fim com maior contato sobre todas as etapas do processo de modelagem, diminuindo assim a atuação do professor que ficará incumbido de conduzir o trabalho e a relação entre os discentes, conforme mencionado em [4], [5] e [7]. Nesse caso o professor pode recomendar quantidade de comércio e de produtos a serem pesquisados para facilitar o resultado final das pesquisas de preço realizada pelos alunos. Por exemplo, o professor poderia sugerir que se pesquisasse o preço de custo e preço de venda de três produtos $\left(P c_{1}, P c_{2}, P c_{3}, P v_{1}, P v_{2}, P v_{3}\right)$ em dois comércios $\left(C_{1}, C_{2}\right)$ da cidade. Tendo assim a organização dos dados tabulados em uma matriz de mesma ordem para todos os alunos. 
Com os alunos de posse desses dados, o professor poderá colocar situações-problema que vão envolver as operações básicas de matrizes(soma, subtração, produto por número real e produto entre matrizes). Questões que tem por objetivo levantar uma discussão em que os alunos identifiquem as variáveis que sejam importantes ao processo e tracem estratégias de resolução. Para uma abordagem mais simples para a primeira questão, indicamos algo que referencie a montagem de uma matriz, como uma questão do tipo "Como podemos organizar os dados coletados para uma melhor leitura dos mesmos?". Dessa maneira o aluno irá experimentar a necessidade de uma ordem nos elementos dessa matriz. Em uma discussão em sala, o aluno perceberá que não haverá somente um único modo de se organizar os dados coletados, mas terá de analisar qual o melhor modo que atenderá a sua necessidade. Tomemos como exemplo, para um melhor entendimento, um possível modelo de organização em tabela:

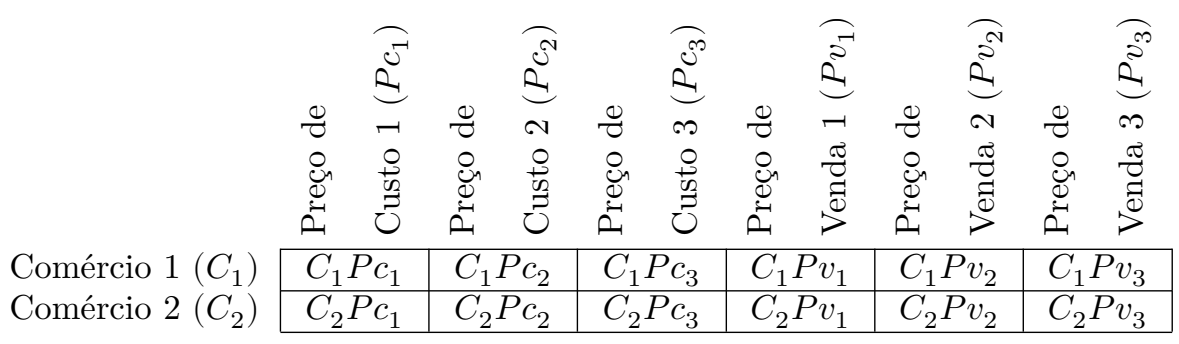

Tabela 1: Distribuição dos valores em relação ao comércio.

que poderá ser compreendida como a uma matriz do tipo $M=\left(a_{i j}\right)_{2 \times 6}$, onde cada coluna indicará o preço do produto e cada linha indicará o comércio onde foi realizada a tomada de preço, conforme a seguir em 1:

$$
M=\left[\begin{array}{llllll}
C_{1} P c_{1} & C_{1} P c_{2} & C_{1} P c_{3} & C_{1} P v_{1} & C_{1} P v_{2} & C_{1} P v_{3} \\
C_{2} P c_{1} & C_{2} P c_{2} & C_{2} P c_{3} & C_{2} P v_{1} & C_{2} P v_{2} & C_{2} P v_{3}
\end{array}\right]
$$

O momento da montagem da matriz de distribuição dos dados é importante, pois o aluno poderá ter um momento de experimentação sobre a localização do "endereço" de cada termo em uma matriz. Compreendendo assim a importância da definição de linhas e colunas de uma matriz e sua organização.

Para uma segunda questão, indicamos algo que sugira soma e subtração de matrizes, que são as operações que oferecem uma facilidade maior para aprendizado por ser necessário uma menor abstração em sua aplicação. O professor poderá usar questões onde sugira soma das matrizes como, por exemplo, "Se uma pessoa comprar uma unidade do produto em cada comércio, quanto gastará?" ou que sugira subtração "Qual o lucro de cada comércio?". Nessa segunda atividade, o aluno perceberá como deve ser o processo de soma e subtração de matrizes, como cada elemento da matriz deve ser somado ou subtraído pelo seu elemento correspondente, desse modo ele mesmo encontrará a resposta à sagaz pergunta "Porque eu não posso somar (ou subtrair) esse primeiro elemento por aquele (ou daquele) último?" que quase sempre surge na classe durante a explicação feita inicialmente pelo professor. Podendo o aluno organizar uma matriz com o valor de venda de cada produto no comércio $1\left(C_{1}\right)$ e outra matriz do comércio $2\left(C_{2}\right)$ e então resolver na forma de soma de matrizes como segue conforme exemplo anterior 


$$
\left[\begin{array}{l}
C_{1} P v_{1} \\
C_{1} P v_{2} \\
C_{1} P v_{3}
\end{array}\right]+\left[\begin{array}{l}
C_{2} P v_{1} \\
C_{2} P v_{2} \\
C_{2} P v_{3}
\end{array}\right]=\left[\begin{array}{l}
C_{1} P v_{1}+C_{2} P v_{1} \\
C_{1} P v_{2}+C_{2} P v_{2} \\
C_{1} P v_{3}+C_{2} P v_{3}
\end{array}\right]
$$

Temos ainda um modelo para subtração de matrizes, o lucro que cada comércio obterá com a venda de cada produto, visto que foram coletados preço de custo e preço de venda. Sendo cabível a indagação "Qual o comércio que terá a o maior lucro com a venda de cada produto?", se fazendo necessária uma subtração entre o valor de venda e o valor de custo para que se determine a matriz de lucro. Que seguindo o modelo anterior, ficará como resultado nos comércios $C_{1}$ e $C_{2}$, respectivamente:

$$
\left[\begin{array}{l}
C_{1} P v_{1} \\
C_{1} P v_{2} \\
C_{1} P v_{3}
\end{array}\right]-\left[\begin{array}{l}
C_{1} P c_{1} \\
C_{1} P c_{2} \\
C_{1} P c_{3}
\end{array}\right]=\left[\begin{array}{l}
C_{1} P v_{1}-C_{1} P c_{1} \\
C_{1} P v_{2}-C_{1} P c_{2} \\
C_{1} P v_{3}-C_{1} P c_{3}
\end{array}\right] \text { e }\left[\begin{array}{l}
C_{2} P v_{1} \\
C_{2} P v_{2} \\
C_{2} P v_{3}
\end{array}\right]-\left[\begin{array}{l}
C_{2} P c_{1} \\
C_{2} P c_{2} \\
C_{2} P c_{3}
\end{array}\right]=\left[\begin{array}{l}
C_{2} P v_{1}-C_{2} P c_{1} \\
C_{2} P v_{2}-C_{2} P c_{2} \\
C_{2} P v_{3}-C_{2} P c_{3}
\end{array}\right]
$$

A terceira atividade que pode ser trabalhada é a multiplicação de uma matriz por um número real, onde o número real é multiplicado por cada elemento da matriz. Podendo ser simulada uma situação onde se quer comprar a mesma quantidade de produtos e se deseja verificar em qual comércio a compra sairá mais em conta, cabendo uma indagação como "Se comprarmos uma quantidade de $n$ unidades de cada produto, em qual comércio o valor a ser pago será menor?". O aluno irá se deparar com uma situação onde terá que multiplicar a matriz por um escalar e ainda fazer uma comparação para verificar se compensa comprar todos os produtos no mesmo comércio ou comprar de modo que, para um menor valor a ser pago, compre produtos nos dois mercados. Modelando essa situação, o aluno chegará ao cálculo matricial

$$
n \cdot\left[\begin{array}{ll}
C_{1} P v_{1} & C_{2} P v_{1} \\
C_{1} P v_{2} & C_{2} P v_{2} \\
C_{1} P v_{3} & C_{2} P v_{3}
\end{array}\right]=\left[\begin{array}{ll}
n \cdot C_{1} P v_{1} & n \cdot C_{2} P v_{1} \\
n \cdot C_{1} P v_{2} & n \cdot C_{2} P v_{2} \\
n \cdot C_{1} P v_{3} & n \cdot C_{2} P v_{3}
\end{array}\right]
$$

$\mathrm{Na}$ quarta atividade podemos trabalhar algo mais complicado que requeira uma familiaridade maior com a soma e produto de números reais. Se colocarmos uma situação que necessite comprar quantidades diferentes de produtos, o aluno teria de traçar uma estratégia mais elaborada para resolver o problema. Nesse caso utilizaria a multiplicação de matrizes percebendo, por si mesmo, as condições necessárias para que possa haver multiplicação entre duas matrizes e o processo prático para efetuá-la. Supondo que seja requerido uma quantidade "a" do produto 1, uma quantidade " $b$ " do produto 2 e uma quantidade " $c$ " do produto 3 , teremos o seguinte modelo para resolução do problema

$$
\left[\begin{array}{lll}
C_{1} P v_{1} & C_{1} P v_{2} & C_{1} P v_{3} \\
C_{2} P v_{1} & C_{2} P v_{2} & C_{2} P v_{3}
\end{array}\right] \cdot\left[\begin{array}{l}
a \\
b \\
c
\end{array}\right]=\left[\begin{array}{l}
a \cdot C_{1} P v_{1}+b \cdot C_{1} P v_{2}+c \cdot C_{1} P v_{3} \\
a \cdot C_{2} P v_{1}+b \cdot C_{2} P v_{2}+c \cdot C_{2} P v_{3}
\end{array}\right]
$$

Que irá mostrar a solução do total a pagar pelos produtos em cada comércio. Um evento interessante da modelagem nessa situação, é a oportunidade do aluno poder se deparar com o erro, 
onde a escolha da ordem da matriz e a disposição de seus elementos, podendo dispor as matrizes conforme alguns exemplos abaixo

$$
\left[\begin{array}{ll}
C_{1} P v_{1} & C_{2} P v_{1} \\
C_{1} P v_{2} & C_{2} P v_{2} \\
C_{1} P v_{3} & C_{2} P v_{3}
\end{array}\right] \cdot\left[\begin{array}{l}
a \\
b \\
c
\end{array}\right],\left[\begin{array}{lll}
C_{1} P v_{1} & C_{1} P v_{2} & C_{1} P v_{3} \\
C_{2} P v_{1} & C_{2} P v_{2} & C_{2} P v_{3}
\end{array}\right] \cdot\left[\begin{array}{lll}
a & b & c
\end{array}\right],\left[\begin{array}{ll}
C_{1} P v_{1} & C_{2} P v_{1} \\
C_{1} P v_{2} & C_{2} P v_{2} \\
C_{1} P v_{3} & C_{2} P v_{3}
\end{array}\right] \cdot\left[\begin{array}{lll}
a & b & c
\end{array}\right]
$$

onde poderá constatar que, para essas matrizes, não existem soluções ou a solução não é interessante para a situação proposta. Chegando por modo empírico á condição necessária e suficiente $n=p$ para que seja possível efetuar a multiplicação entre as matrizes $\left(a_{i j}\right)_{m \times n} \times\left(b_{i j}\right)_{p \times q}$.

Na quinta proposta de atividade, consideramos uma abordagem onde se necessite de soma de matrizes e multiplicação por um número natural. Podemos tomar como exemplo uma questão do modelo "Se no comércio onde os produtos são mais baratos não tiver quantidade suficiente, quanto custará se comprarmos o restante dos produtos necessários no outro comércio?". Supondo que necessitamos de uma quantidade $y$ de cada produto e no comércio onde os produtos têm o menor preço tenha somente uma quantidade $z$ de cada produto e seja necessário comprar também uma quantidade $t$ de produtos no outro comércio, onde $y=z+t$. Teremos um modelo matemático no molde

$$
\left[\begin{array}{l}
C_{1} P v_{1} \\
C_{1} P v_{2} \\
C_{1} P v_{3}
\end{array}\right]+t \cdot\left[\begin{array}{l}
C_{2} P v_{1} \\
C_{2} P v_{2} \\
C_{2} P v_{3}
\end{array}\right]=\left[\begin{array}{l}
z \cdot C_{1} P v_{1} \\
z \cdot C_{1} P v_{2} \\
z \cdot C_{1} P v_{3}
\end{array}\right]+\left[\begin{array}{l}
t \cdot C_{2} P v_{1} \\
t \cdot C_{2} P v_{2} \\
t \cdot C_{2} P v_{3}
\end{array}\right]=\left[\begin{array}{l}
z \cdot C_{1} P v_{1}+t \cdot C_{2} P v_{1} \\
z \cdot C_{1} P v_{2}+t \cdot C_{2} P v_{2} \\
z \cdot C_{1} P v_{3}+t \cdot C_{2} P v_{3}
\end{array}\right],
$$

onde o aluno perceberá que operações com matrizes mantêm as regras de operação da aritmética usual (com exceção da comutatividade multiplicativa!) Nesse caso opera-se inicialmente com a multiplicação e posteriormente a adição (ou subtração) entre as matrizes. Pode-se ainda obter um último modelo de situação-problema que é ter quantidades distintas de cada produto sob a indagação "Se no comércio onde os produtos são mais baratos não tiver quantidades suficientes, quanto custaria se comprarmos o restante dos produtos necessários em outro comércio?" que recorreremos à multiplicação e soma de matrizes. Para tanto definiremos a cargo de exemplo as quantidades dos produtos 1,2 e 3 no comércio $1 C_{1}$ como, respectivamente, $a, b$ e $c$ e as quantidades dos produtos 1,2 e 3 no comércio $2 C_{2}$ como, respectivamente, $d$, e e $f$ cujo modelo matemático se dará no modo seguinte da equação matricial 7

$$
\left[\begin{array}{lll}
C_{1} P v_{1} & C_{1} P v_{2} & C_{1} P v_{3}
\end{array}\right] \cdot\left[\begin{array}{l}
a \\
b \\
c
\end{array}\right]+\left[\begin{array}{lll}
C_{2} P v_{1} & C_{2} P v_{2} & C_{2} P v_{3}
\end{array}\right] \cdot\left[\begin{array}{l}
d \\
e \\
f
\end{array}\right]
$$

que resolvendo as multiplicações através do algoritmo da multiplicação visto anteriormente, teremos a equação matricial 8, onde a primeira parcela é o valor pago no comércio $1\left(C_{1}\right)$ e a segunda parcela é o valor pago ao comércio $2\left(C_{2}\right)$

$$
\left[a \cdot C_{1} P v_{1}+b \cdot C_{1} P v_{2}+c \cdot C_{1} P v_{3}\right]+\left[d \cdot C_{2} P v_{1}+e \cdot C_{2} P v_{2}+f \cdot C_{2} P v_{3}\right]
$$

que finalmente resultará no valor total pago nos comércios 1 e 2 como na matriz 9 


$$
\left[a \cdot C_{1} P v_{1}+b \cdot C_{1} P v_{2}+c \cdot C_{1} P v_{3}+d \cdot C_{2} P v_{1}+e \cdot C_{2} P v_{2}+f \cdot C_{2} P v_{3}\right]
$$

Chegando assim ao final do caminho a ser percorrido pelos alunos à descoberta do conhecimento de matrizes. Perpassando essas questões, o aluno terá adquirido conhecimento para perceber a utilização das operações básicas usuais nos livros didáticos utilizados na escola.

\section{Metodologia}

Ao lançarmos mão do trabalho de investigação e pesquisa in loco, junto ao grupo composto por 34 (trinta e quatro) alunos do 2 o ano turma "A" do ensino médio da Escola Estadual de Ensino Médio José Luiz Martins, no município de Água Azul do Norte no estado do Pará. Essa turma foi escolhida por ser formada por um alunado bastante diversificado, nessa turma estudam alunos tanto da zona rural quanto da zona urbana, alunos do sexo feminino e masculino e de famílias de diversos ramos de atividades, de pequenos lavradores à empresários dessa pequena cidade. Essas várias situações se culminam nessa turma devido essa ser a única escola de ensino médio da cidade.

Abrimos os caminhos rumo à pesquisa para o bem estar dos participantes, caracterizando e estruturando uma investigação junto ao comércio local, cujo os segmentos de atuação são diferenciados, sendo sua realização delimitada em no mínimo 2 (dois) e no máximo 3 (três) estabelecimentos comerciais. A proposta foi orientada para 16 horas aulas referente ao estudo de matrizes, conteúdo que integra a grade curricular da classe de alunos em destaque. Na perspectiva de averiguarem e estruturarem em formas de tabelas, os valores de venda e compra, por parte do comerciante e dos consumidores, um mínimo de 2 (dois) e um máximo de 3 (três) produtos. A vertente deste trabalho circula em torno de 6 (seis) questões que são os problemas da pesquisa a ser desenvolvida pelos estudantes.

São elas:

1) Como podemos organizar os dados coletados para uma melhor leitura dos mesmos?

2) Se uma pessoa comprar uma unidade do produto em cada comércio, quanto gastaria?

3) Qual o comércio que teria a o maior lucro com a venda de cada produto?

4) Se comprarmos uma quantidade de $n$ unidades de cada produto, em qual comércio o valor a ser pago seria menor?

5) Supondo que seja requerido uma quantidade " $a$ " do produto 1 , uma quantidade " $b$ " do produto 2 e uma quantidade " $c$ " do produto 3 , em qual comércio o preço seria menor?

6) Se no comércio onde os produtos são mais baratos não tiver quantidades suficientes, quanto custaria se comprarmos o restante dos produtos necessários em outro comércio?

No decorrer das aulas, os professores autores, desenvolveram de forma tradicional os conceitos de matrizes, com aulas expositivas e resolução de exercícios para melhor relacionar e fixar o conteúdo ensinado. Porém, cada dupla de alunos já havia coletado suas informações junto ao comercio local, esse modelo de aula inquietou os alunos, pois, os mesmos ainda não conseguiam relacionar a pesquisa feita com o conteúdo estudado. Dessa forma, ao apresentar as 6 (seis) questões acima citadas, tornou-se mais visível a relação entre a modelagem e o conteúdo abordado.

Ao ser pedido para organizarem os dados adquiridos em tabelas estruturadas, dados esses que estavam soltos e sem nenhuma forma, tornaram-se objetos plausíveis para resolução dos problemas propostos. Facilitando o trabalho dos estudantes-pesquisadores que já relacionavam a Matemática ali empregada com o trabalho de "pechincha", palavra comum na linguagem regional que significa tomada de preços, que suas mães faziam ao escolherem os melhores comércios para fazerem suas compras e os produtos mais em conta.

A metodologia aqui empregada é a pesquisa exploratória que de acordo com as ideias de [1], [14] e [11], corrobora na investigação a respeito do tema abordado, pois normalmente o tema é pouco conhecido, 
ajudando o pesquisador a obter uma melhor visão e um maior aprofundamento do assunto pesquisado, possibilitando a formulação de hipóteses para melhor exploração do objeto investigado.

Segundo [7] essa é a metodologia usual da tendência em educação Matemática denominada Modelagem Matemática ou simplesmente Modelagem, pois a(s) problemática(s) abordada(s) pela Modelagem, sempre $\operatorname{apresenta}(\mathrm{m})$ características diferenciadas das problemáticas convencionais, normalmente encontrada nos livros didáticos, pois os problemas que a Modelagem aborda, nascem da pesquisa e coleta de dados, tanto qualitativa quanto quantitativa, que é decorrente da pesquisa exploratória.

As explicações em torno dos conceitos de matrizes, em nossa percepção, em relação aos alunos, tornaramse mais consistentes, pois os mesmos não assimilavam tais conceitos teóricos com sua aplicabilidade em diversos ramos das ciências, e a pesquisa exploratória tornou visível esse intercambio entre a matemática e as demais ciências, com o seu cotidiano, desde o mais simples ao mais complexo.

Notamos que a proposta apresentada segue certo padrão, porém, nada impede que o professor incremente suas aulas com novas hipóteses ou até mesmo, surjam novos e importantes questionamentos entre os estudantes.

\section{Considerações Finais}

Dar enfoque a situações-problemas reais nas aulas de Matemática permite-nos uma maior aproximação de um saber Matemático com absoluta significância para os estudantes, pois o aluno torna-se investigador, pesquisador, fazendo parte do desenvolvimento e da aplicação dos dados, gerando maior interesse, entusiasmo e motivação pelas aulas, notando que a Matemática se faz presente no nosso dia-a-dia.

Os conteúdos têm distintas aplicabilidades e é necessário revelar-lhes aos alunos, como forma de cooperação para a seu desenvolvimento integral para a vida e para o mercado de trabalho. Assim, observamos inicialmente a insatisfação dos alunos com as aulas e ao adquirem esse novo olhar, novos ânimos estimularam os estudantes e um clima agradável tomou conta do ambiente educacional ao desenvolvermos as atividades através da Modelagem.

O bom emprego de situações reais com o desenvolvimento do conteúdo de matrizes para a interpretação e a criticidade, matematicamente falando, em aulas bem elaboradas, aguça a visão dos alunos ao quanto a Matemática é fundamental e se faz presente no nosso cotidiano. Através da Modelagem, os mesmos assumem o papel de gestão do problema dado, tomando decisões importantes à resolução do problema. Revelando-os que tais decisões respeitam certos critérios e seguem certo rigor, destacando a Matemática como ferramenta fundamental para a resolução de tal problema.

Portanto, esperamos que a prática desta metodologia junto às aulas de Matemática possa além de servir como ferramenta motivadora, também contribuir introduzindo novos enfoques, propiciando a compreensão e interpretação dos problemas reais vividos pelos estudantes no meio ao qual o mesmo encontra-se inserido e faça também com que ele perceba o quanto ele está inserido e que tudo faz parte do processo de cidadania.

Deste modo, o ensino da Matemática exerce seu papel de colaborar na constituição do indivíduo, abordando assuntos e questões do cotidiano, sendo instrumento de investigação e compreensão com o intuito de revelar, avaliar e deliberar o mais exato possível.

\section{Referências}

[1] Andrade, M. M. de. Como Preparar Trabalhos para Cursos de Pós-Graduação: Noções Práticas. 2002.

[2] Ausubel, D. P.; Novak, J. D.; Hanesian, H. Psicologia Educacional. Segunda edição. Rio de Janeiro: Interamericana, 1980. 
[3] Ausubel, D. P. Aquisição e Retenção de Conhecimentos: Uma Perspectiva Cognitiva. Editora Plátano, 2003.

[4] Barbosa, J. C. Modelagem na Educação Matemática: Contribuições para O Debate Teórico. Reunião anual da ANPED, v. 24, p. 1-15, 2001.

[5] Barbosa, J. C. Modelagem Matemática em Sala de Aula. Perspectiva, v. 27, n. 98, 2003.

[6] Bassanezi, C. B. Ensino-Aprendizagem com Modelagem Matemática: Uma Nova Estratégia. Contexto, 2002.

[7] Burak, D. Modelagem Matemática e a Sala de Aula. In: I EPMEM - Encontro Paranaense da Modelagem Na Educação Matemática. Londrina. Anais do I EPMEM, 2004.

[8] Correa, J.; McLean, M. Era Uma Vez... Um Vilão Chamado Matemática: Um Estudo Intercultural da Dificuldade Atribuída À Matemática. Universidade Federal do Rio Grande do Sul, 1999.

[9] D’ambrosio, U. Etnomatemática. São Paulo: Àtica, 1998.

[10] Ferreira, D. H. L.; Wodewotzki, M. L. L. Modelagem Matemática e Educação Ambiental: Uma Experiência com Alunos do Ensino Médio: Artigo Científico. PUC-Campinas, 2005.

[11] Gil, A. C. Métodos e Técnicas de Pesquisa Social. In: Métodos e técnicas de pesquisa social. Atlas, 2010.

[12] Messias, M. A. de V. F.; de Sá, P. F.; Fonseca, R. V. Um Estudo Diagnóstico sobre as Dificuldades em Matrizes. IX ENEM - Encontro Nacional de Educação Matemática, Belo Horizonte, MG, 2007. Disponível em: <http://www.sbem.com.br/files/ix_enem/Poster/Trabalhos/ PO83969659272T.doc $>$. Acesso em: 20 de agosto de 2014.

[13] Pelizzari, A.; Kriegl M. de L.; Baron, M. P.; Fink, N. T. L.; Dorocinski, S. I. Teoria da Aprendizagem Significativa Segundo Ausubel. Revista PEC, v. 2, n. 1, p. 37-42, 2002.

[14] Raupp, F. M.; Beuren, I. M. Metodologia da Pesquisa Aplicável Às Ciências Sociais. Como Elaborar Trabalhos Monográficos em Contabilidade: Teoria e Prática, v. 3, p. 76-97, 2003.

[15] Silva, L. M. da. A Ficção e o Ensino da Matemática: Análise do Interesse de Estudantes em Resolver Problemas. 2014. Disponível em: <http://meriva.pucrs.br/dspace/bitstream/10923/ 6686/1/000459164-Texto\%2BCompleto-0.pdf >. Acesso em: 20 de agosto de 2014.

Ademir Costa

Secretaria de Educação do Estado do Pará (SEDUC/PA) <ademirbrandao@gmail.com>

Thiago Lopes

Instituto Federal do Mato Grosso (IFMT) $<$ thiagobeirigolopes@yahoo.com.br>

Recebido: 2015

Publicado: 2015 